\title{
Functional MRI, DTI and neurophysiology in horizontal gaze palsy with progressive scoliosis
}

\author{
Sven Haller • Stephan G. Wetzel • Jürg Lütschg
}

Received: 19 August 2007 / Accepted: 14 December 2007 / Published online: 24 January 2008

(C) Springer-Verlag 2008

\begin{abstract}
Introduction Horizontal gaze palsy with progressive scoliosis ascending lemniscal and descending corticospinal tracts in the systems.

Methods We evaluated the use of functional magnetic resonance imaging (fMRI) for the first time in this disease and compared it to diffusion tensor imaging (DTI) tractography and neurophysiological findings in the same patient with genetically confirmed $\mathrm{ROBO} 3$ mutation.

Results As expected, motor fMRI, somatosensory evoked potentials (SSEP) and motor evoked potentials (MEP) were dominantly ipsilateral to the stimulation side. DTI tractography revealed ipsilateral ascending and descending connectivity in the brainstem yet normal interhemispheric connections in the corpus callosum. Auditory fMRI revealed bilateral auditory activation to monaural left-sided auditory stimulation. No significant cortical activation was observed after monaural right-sided stimulation, a hearing defect having been excluded. Prosaccades fMRI showed no activations in the eye-movement network.

Conclusion Motor fMRI confirmed the established findings of DTI and neurophysiology in the same patient. In (HGPPS) is an autosomal recessive disease due to a mutation in the $\mathrm{ROBO} 3$ gene. This rare disease is of particular interest because the absence, or at least reduction, of crossing of the medulla predicts abnormal ipsilateral sensory and motor suspected HGPPS, any technique appears appropriate for further investigation. Auditory fMRI suggests that a monaural auditory system with bilateral auditory activations might be a physiological advantage as compared to a binaural yet only unilateral auditory system, in analogy to anisometropic amblyopia. Moving-head fMRI studies in the future might show whether the compensatory head movements instead of normal eye movements activate the eyemovement network.

Keywords Functional MRI $\cdot$ HGPPS $\cdot$ ROBO3

$\begin{array}{ll}\text { Abbreviations } \\ \text { BAEP } & \text { brainstem auditory evoked potentials } \\ \text { BOLD } & \text { blood oxygenation level dependent } \\ \text { DTI } & \text { diffusion tensor imaging } \\ \text { fMRI } & \text { functional magnetic resonance imaging } \\ \text { FEF } & \text { frontal eye field } \\ \text { HGPPS } & \text { horizontal gaze palsy with progressive scoliosis } \\ \text { MEP } & \text { motor evoked potentials } \\ \text { MT/MST } & \text { medial temporal cortex } \\ \text { PEF } & \text { parietal eye field } \\ \text { ROI } & \text { region of interest } \\ \text { SEF } & \text { supplementary eye field } \\ \text { SMA } & \text { supplementary motor area } \\ \text { SSEP } & \text { somatosensory evoked potentials }\end{array}$

\section{Introduction}

Horizontal gaze palsy with progressive scoliosis (HGPPS) is characterized by congenital absence of horizontal gaze and progressive scoliosis [1]. This autosomal recessive disease is associated with at least ten different mutations in 
the human $R O B O 3$ gene located at chromosome 11q23-25 $[2,3]$ that is critical for the crossing of long ascending medial lemniscal and descending corticospinal tracts in the medulla $[4,5]$. Characteristic conventional neuroimaging findings consist of a hypoplastic pons and cerebellar peduncles as well as anterior and posterior clefts of the pons and medulla oblongata $[2,4,5]$. This rare disease is of particular interest because it suggests abnormal ipsilateral instead of the normal contralateral sensory and motor pathways. This was demonstrated neuroradiographically by diffusion tensor imaging (DTI) [4] and electrophysiologically by somatosensory evoked potentials (SSEP) and motor evoked potentials (MEP) $[2,5,6]$, or intraoperative neurophysiology [7]. No study has combined DTI and electrophysiology in the same patient.

We used functional magnetic resonance imaging (fMRI) for the first time in this disease and implemented a basic motor task, a basic auditory task and a prosaccades task. The findings were compared to those from DTI, SSEP and MEP in the same patient with HGPPS due to a genetically confirmed ROBO3 mutation.

\section{Methods}

Patient history and neurological examination

A 14-year-old boy presented with the clinical picture of HGPPS with genetically confirmed ROBO3 mutation $(2108 \mathrm{G}>\mathrm{C} ; \mathrm{R} 703 \mathrm{P})$ and no history of scoliosis or gaze palsy in other members of the family. The patient's birth was normal, and he was born in Kosovo. His psychomotor development had been normal except for marked gaze fixation, which was noticed at the age of 6 months. From the age of 2 years he showed jerking head movements and some gait instability, but no visual problems. At the age of 7 years, scoliosis was observed. He attended a normal school where his attainment was good and he was able to participate in gymnastics lessons.

On initial admission to our hospital neurological examination revealed a complete loss of horizontal eye movements whereas vertical eye movements were normal. Convergence movements were also markedly reduced. Caloric stimulation with ice water failed to evoke horizontal deviation in either eye. There was only moderate vertical nystagmus detectable and no abnormalities of the other cranial nerves including hearing were seen. In addition he showed a moderate intention tremor and unsteadiness on standing or jumping on one leg. There was also moderate scoliosis with a Cobb angle of $20^{\circ}$. The essential clinical presentation, notably the horizontal gaze palsy, the strongly reduced convergence and the scoliosis, is illustrated in Fig. 1.

\section{Structural MRI}

Structural MRI scans were obtained in a 1.5-T routine clinical MR scanner. Axial and coronal fast spin echo T2weighted images (matrix 512 $\times 512$, 5-mm slice thickness, TE $109 \mathrm{~ms}$, TR 4,900 ms) were recorded.
Fig. 1 Clinical presentation: a complete absence of horizontal gaze; $\mathbf{b}$ convergence greatly reduced; c, d vertical eye movements within normal limits; e moderate scoliosis

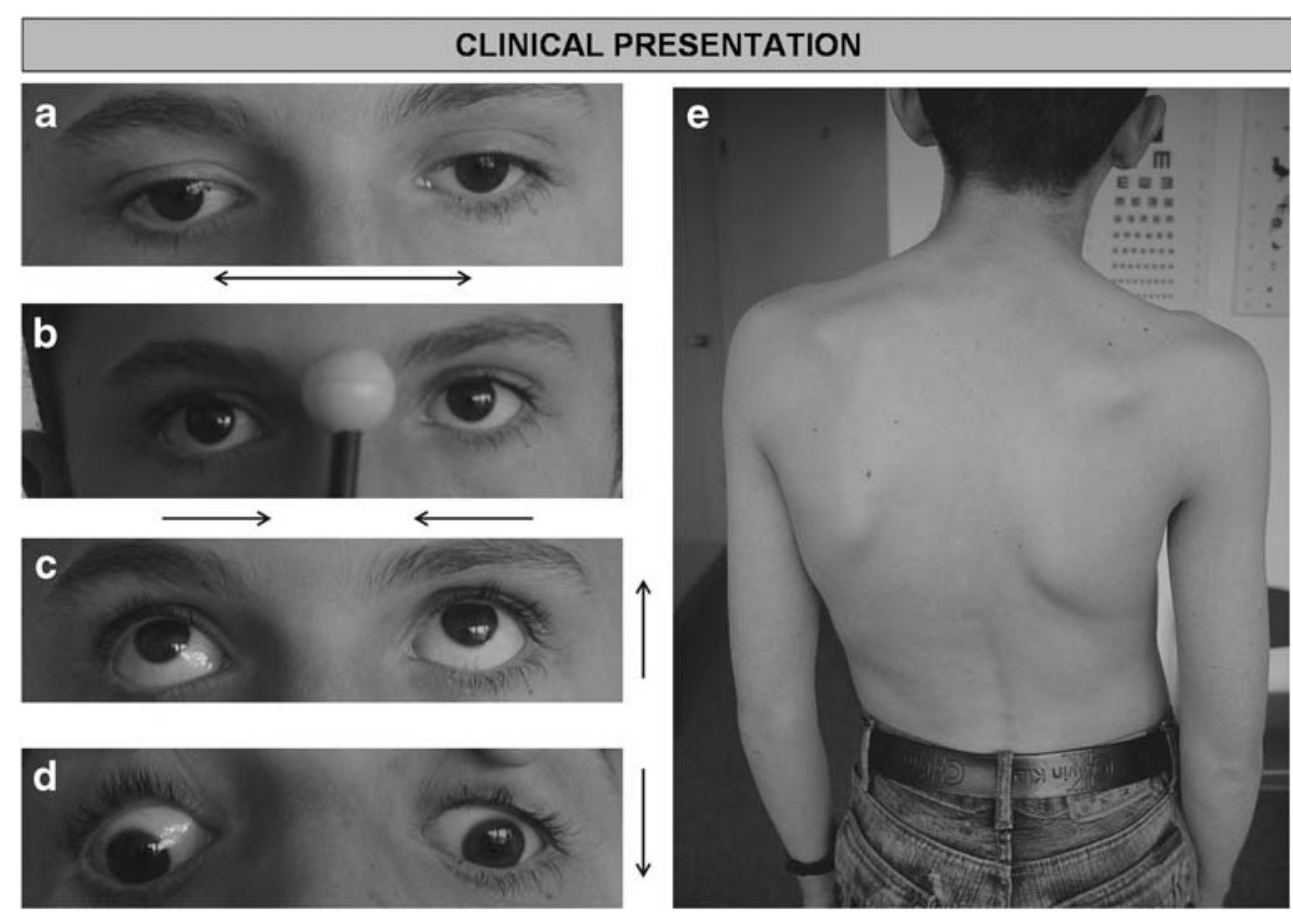




\section{Functional MRI}

Functional MR imaging was performed on a whole-body routine clinical 1.5-T scanner with a 12-channel head coil. Three experimental tasks were performed. In the motor (finger-tapping) task, the patient was instructed to move either the right or left finger with rest periods according to a visual stimulus in a block design with the following periods: left finger (20 s), rest (10 s), right finger (20 s) and rest $(10 \mathrm{~s})$. This cycle was repeated six times. In the auditory task, a sine tone of $1000 \mathrm{~Hz}$ pulsating at $6 \mathrm{~Hz}$ that was presented to induce a strong and long-lasting BOLD response was used [8]. This auditory stimulus was presented monaurally to the right ear in the first run and monaurally to the left ear in the second run in a block design with $20 \mathrm{~s}$ ON and 20 s OFF, with nine repetitions. The prosaccades task consisted of a block design with eight alternating blocks of ON (20 s) and OFF (20 s). Two runs were performed. Targets were randomly presented to the far right of the possible visual field (equivalent to $11.5^{\circ}$ ), to the intermediate right $\left(5.75^{\circ}\right)$, to the intermediate left or to the far left on average every $500 \mathrm{~ms}$ (jittered $\pm 100 \mathrm{~ms}$ ).

For the motor and prosaccades tasks, functional $\mathrm{T} 2 *_{\text {- }}$ weighted images were obtained based on a conventional echo-planar single-shot (EPI) pulse sequence: matrix size $64 \times 64$, field of view (FOV) $210 \times 210 \mathrm{~mm}, 28$ slices of 4 $\mathrm{mm}$ thickness, 1-mm gap covering the whole brain, flip angle (FA) $90^{\circ}$, repetition time (TR) $2.5 \mathrm{~s}$, echo time (TE) $50 \mathrm{~ms}, 147$ repetitions (motor) or 131 repetitions (prosaccades). For the auditory task, an EPI sequence with a novel modification with continuous rather than pulsed EPI background noise, which substantially increased the BOLD response to basic auditory stimuli [9], was implemented. Imaging parameters were: matrix size $64 \times 64$, FOV $210 \times 210 \mathrm{~mm}, 27$ slices of 4-mm thickness, 1-mm gap, FA 90, TR $2.97 \mathrm{~s}, \mathrm{TE} 61 \mathrm{~ms}, 121$ repetitions. Additionally, a high-resolution 1-mm isovoxel T1-weighted magnetization-prepared rapid gradient echo (MPRAGE) was acquired (matrix 256×256, 176 sections).

Anatomical and functional images were analyzed using the BrainVoyager QX software package (Brain Innovation, Maastricht, The Netherlands). Preprocessing of the functional time series consisted of three-dimensional motion correction, interscan slice timing correction, gaussian spatial filtering (full width half maximum $4 \mathrm{~mm}$ ), highpass temporal filtering (three cycles per time course) and transformation into standard space [10]. The statistical analysis was implemented as separate general linear models (GLM) for the different tasks described above. Statistical thresholding was corrected for multiple comparisons based on the false discovery rate (FDR) [11] at a false-positive probability of $\mathrm{q}(\mathrm{FDR})<0.001$ for the motor task (corresponding to $t>4.3$ ) and of $\mathrm{q}(\mathrm{FDR})<0.01$ for the auditory and prosaccades task (corresponding to $t>4.3$ and $t>3.8$, respectively).

\section{DTI tractography}

The DTI protocol was: matrix size $256 \times 256$, FOV $250 \times$ $250 \mathrm{~mm}, 24$ slices of 5-mm thickness, $0 \mathrm{~mm}$ gap, TR $4.2 \mathrm{~s}$, TE $110 \mathrm{~ms}$, six directions. Two volumes were acquired. The cranial volume covered the motor cortex, and the caudal volume covered the medulla oblongata. DTI tractography was analyzed using the MGH diffusion tensor imaging toolbox (http://www.nmr.mgh.harvard.edu/ $\sim$ rpwang/siemens/dti_taskcard/new/). In the caudal volume, bilateral volumes of interest were defined in the brainstem. In the cranial volume, bilateral volumes of interest were defined in bilateral motor areas and additionally in the corpus callosum.

\section{Neurophysiology}

MEPs were obtained using a magnetic stimulator with a circular magnetic coil with an outside diameter of $14 \mathrm{~cm}$. The center of the coil was placed $2 \mathrm{~cm}$ posterior and $2-$ $3 \mathrm{~cm}$ lateral to electrode $\mathrm{Cz}$ to stimulate the finger area. Surface recording electrodes were placed bilaterally on the abductor digiti $\mathrm{V}$ muscles (recording device Nicolet Viasys). SSEPs were elicited by stimulation of the right and left median nerve (electrical square wave pulses of $0.1 \mathrm{~ms}$ duration) at the wrist. A total of 250 responses were averaged. The potentials were recorded simultaneously over the right and left parietal region $(2 \mathrm{~cm}$ behind $\mathrm{C} 3$ and $\mathrm{C} 4$ and reference electrode over Fpz). Brainstem auditory evoked potentials (BAEP) were elicited by clicks of $0.1 \mathrm{~ms}$ duration and $70 \mathrm{~dB}$ HL sound intensity. They were recorded from the vertex $(\mathrm{Cz})$ and the ipsilateral mastoid (A1 or A2). The screen time was $20 \mathrm{~ms}$.

\section{Results}

\section{Structural MRI}

Structural MRI demonstrated a hypoplastic pons and medulla oblongata. There was a complete midline cleft at the level of the medulla oblongata that continued cranially as a partial, dorsal midline cleft throughout the pons to the level of the fourth ventricle (Fig. 2).

\section{Functional MRI}

We performed two runs of the motor and prosaccades tasks. The corresponding activation maps were very similar, implying high reliability of the recorded data. The motor 
Fig. 2 Axial T2-weighted slices demonstrate the hypoplastic pons and medulla with a prominent midline cleft and the resulting characteristic "butterfly" configuration

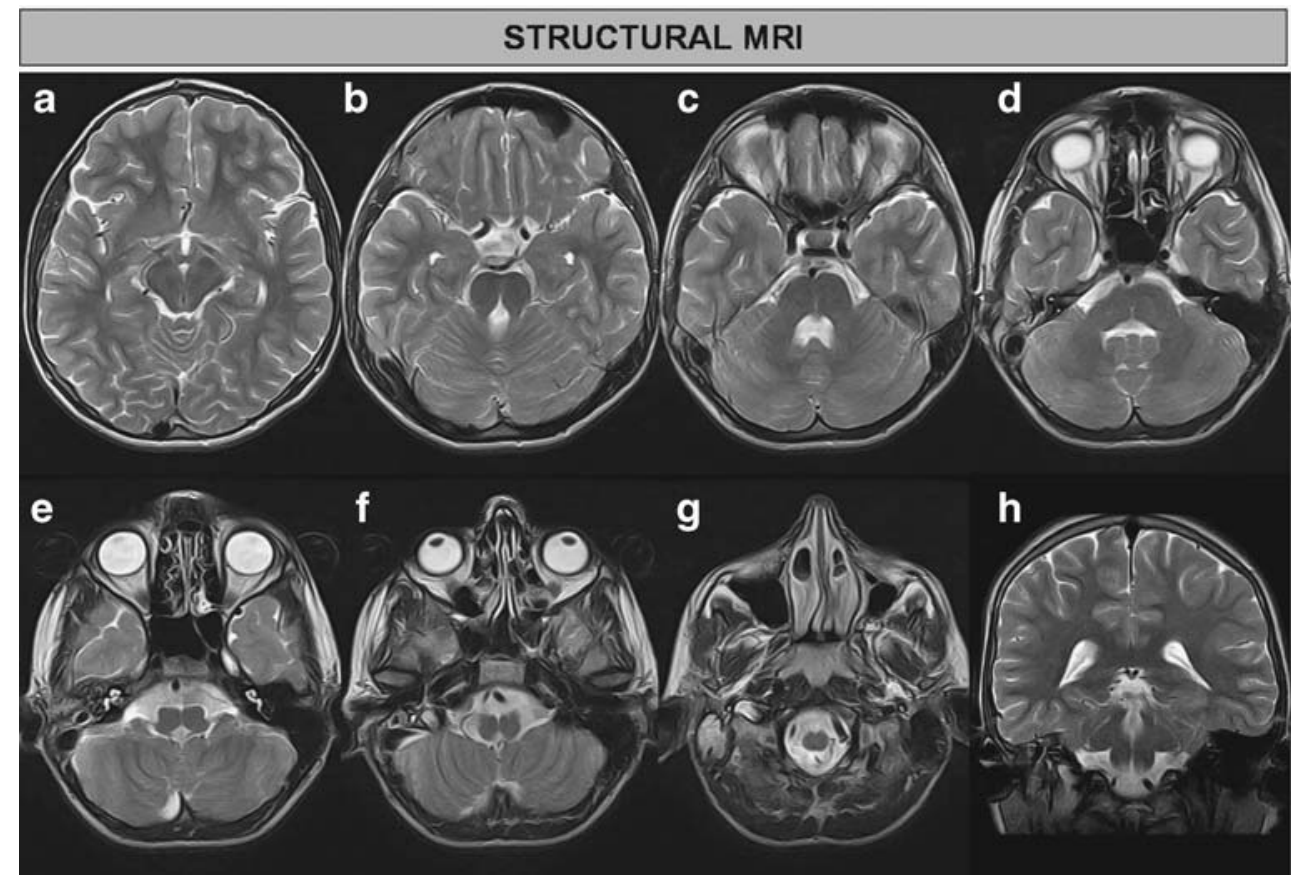

task evoked ipsilateral activation in the primary motor and sensory cortex in contrast to that in the presence of normal anatomy where contralateral activation would be expected (Fig. 3a). Left-sided monaural auditory stimulation evoked bilateral activations in the primary auditory area, while right-sided monaural stimulation evoked no significant auditory activations (Fig. 3b).The prosaccades task evoked no activations in the high frontal and parietal areas housing the functional areas supplementary motor area (SMA) frontal eye fields (FEF) and parietal eye fields (PEF). Bilateral activation was present at the occipitotemporal junction (Fig. 3c).

\section{DTI tractography}

The bilateral ROIs in the lower brainstem showed only ipsilateral ascending and descending connectivity (Fig. 4ac). The ROIs in the bilateral primary motor area also showed only ipsilateral descending tracts with ipsilateral connectivity to the cerebellum (Fig. 4d,e). None of the above-mentioned ROIs showed midline-crossing fibers. In contrast, the ROI in the corpus callosum showed the normal interhemispheric connections (Fig. 4f).

\section{Neurophysiology}

The MEPs evoked by magnetic pulses over both centroparietal regions elicited only ipsilateral responses (Fig. 5a). The latency of the potentials was within normal limits. The SSEP elicited by stimulation of the median nerve showed an ipsilateral N20 response of normal latency and amplitude on both sides and there was a very small contralateral response (Fig. 5b). The small contralateral response seen in the SSEP with the recording sensitivity used represented volume conduction from the ipsilateral response as has been shown previously [12]. The BAEPs were normal after stimulation of the left ear (latency of wave I $1.8 \mathrm{~ms}$, wave III $4.1 \mathrm{~ms}$ and wave V $5.6 \mathrm{~ms}$ ) but abnormal after rightsided stimulation (wave I latency $2.4 \mathrm{~ms}$, wave III $4.5 \mathrm{~ms}$ and wave V $6.2 \mathrm{~ms}$; not illustrated).

\section{Discussion}

The absence, or at least severe reduction, of midline crossing fibers at the level of the medulla in HGPPS is of particular interest because it suggests abnormal ipsilateral instead of normal contralateral sensorimotor networks [2, $3]$. Compared to previous investigations $[2,4,5]$, we provide two new aspects. First, we assessed fMRI for the first time in a patient with genetically confirmed ROBO3 mutation and implemented not only a motor, but also an auditory and a prosaccade task. Second, we additionally assessed SSEP, MEP and DTI tractography in the same patient. These methods have been applied previously, but in different patients.

All findings related to the sensorimotor system, yielded consistent results with dominantly ipsilateral fMRI motor activations in the primary sensorimotor area, ipsilateral SSEPs and MEPs, and ipsilateral ascending and descending tractography without crossing fibers at the level of the brainstem. These results are consistent with those of previous investigations [2, 4, 5]. In contrast, another previous investigation found partial crossing of a minority 


\section{FUNCTIONAL MRI}
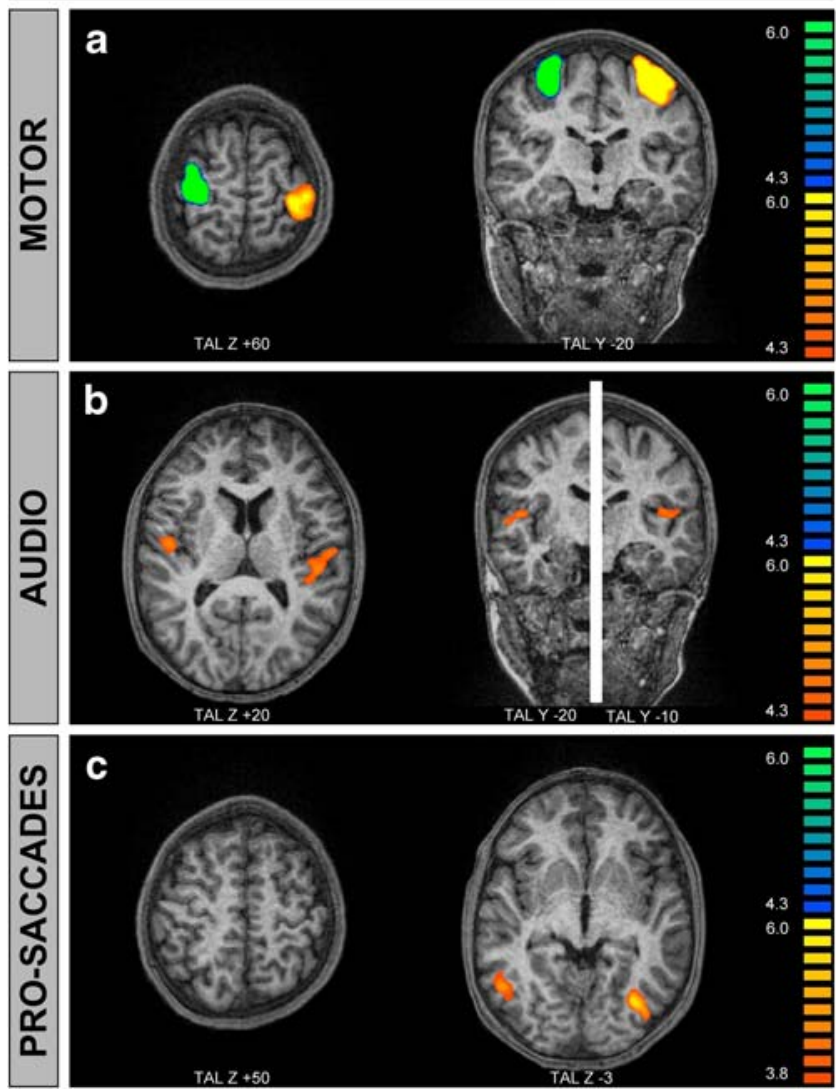

Fig. 3 Structural MRI, BOLD activations. a In the motor task, leftsided finger movement evokes activation in the ipsilateral left primary sensorimotor area (red-yellow) while right-sided movement elicited activations in ipsilateral right primary sensorimotor area (blue-green). b In the auditory task, only left-sided auditory activation evokes bilateral auditory activations (red-yellow) but right-sided stimulation yields no suprathreshold activations. c In the prosaccades task, no suprathreshold activations are present in the saccade network including SMA, SEF, FEF and PEF. Activations are present in the occipitotemporal junction that may be considered as human homologue of MT/MST

of connectivities [13]. Additionally, a partial crossing of the dorsal column-medial lemniscal system was observed in one patient [6]. Note that DTI tractography depends on the selected ROI and tractography algorithm. Furthermore, DTI tractography is not sensitive enough to detect minor crossings. Taking all investigations together, at least the majority of connectivities are ipsilateral. DTI tractography of the corpus callosum provided evidence of normal interhemispheric fibers at the level of the cerebrum. This is in line with the isolated defect of fiber crossing in the brainstem, whereas fibers crossing in other areas of the brain, for example the interhemispheric connections in the corpus callosum, are intact. The consistent results of the different modalities support their validity, but the combination of these modalities in the same patient yielded no added value. On the other hand, this suggests that if HGPPS is suspected, any of these techniques - the least expensive or most readily available - can be performed.

The reported patient showed a complete absence of horizontal eye movements, severely reduced convergence and progressive scoliosis. In the series of Bosley et al. [5], absent horizontal eye movements were seen in all patients but convergence was minimal in 3 of 11 patients but excellent in 5 of 11 patients Therefore it can be assumed just from the clinical findings that the midline defect, as indicated by the "butterfly" appearance of the brainstem, may be variable. Unfortunately, detailed anatomic information is not available in the above-mentioned study. Variations in the extent of the split brainstem may explain why in some cases the convergence is normal and in others it is abolished. We performed fMRI in order to further investigate the neuronal network associated with eye movement. The reason for performing the prosaccade task despite the known horizontal gaze palsy originated from previous experience in patients with other defects such as aphasia, in whom activations in language areas may be observed despite clinical aphasia.

The prosaccade task elicited no activations in the established saccade network including the SMA, bilateral FEF and PEF [14-16]. These finding are remarkable because FEF/PEF are usually also activated by attention shifts even without gaze shifts [19]. The patient was instructed to follow the target dot. No specific instructions with respect to attention were given. He reported that he perceived the moving dot. Correspondingly, bilateral activation in the occipitotemporal junction was present. This area is considered to be the human homologue of the monkey MT/MST system [15, 17, 18]. As the patient perceived the moving stimulus, it is very likely that he also performed "normal" or involuntary shifts of attention. These results should be interpreted with great caution as this is only a single case report and we did not control eye movements (e.g. MR compatible eye-tracker). Nevertheless, these findings might support the similarities between the neuronal circuitries that mediate gaze shifts and attention shifts. Both systems appeared to be impaired in the patient. Because of the horizontal gaze palsy, the patient performed compensatory head movements. Unfortunately, fMRI requires a stable head position and consequently could not determine whether these compensatory horizontal head movements were associated with activations in the eye-movement network. However, current development in fMRI might allow investigation of the moving head in the near future. It would be of particular interest to determine whether head movements performed to compensate for horizontal gaze palsy activate the established eye-movement network.

The most interesting result of the fMRI investigation originates from the auditory task. In normal volunteers, 
Fig. 4 DTI tractography. Bilateral ROIs in the lower brain stem $(\mathbf{a}, \mathbf{b}, \mathbf{c})$ demonstrate ipsilateral tracts superimposed on axial $(\mathbf{a}, \mathbf{b})$ and coronal (c) sections. Bilateral ROIs in the primary motor area $(\mathbf{d}, \mathbf{e}$, axial sections) also demonstrate only ipsilateral descending tracts. Note the ipsilateral cerebellar tracts. The presence of crossing fibers is demonstrated by the ROI in the corpus callosum (f)

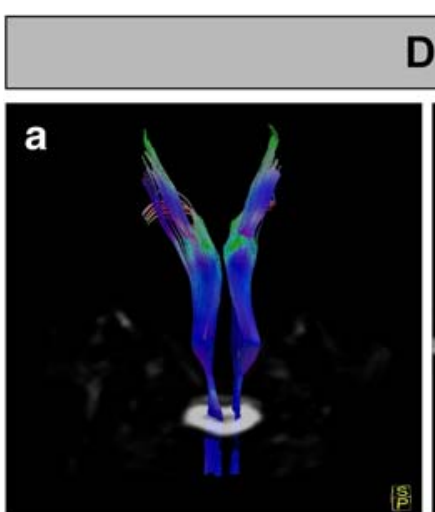

\section{DTI - TRACTOGRAPHY}
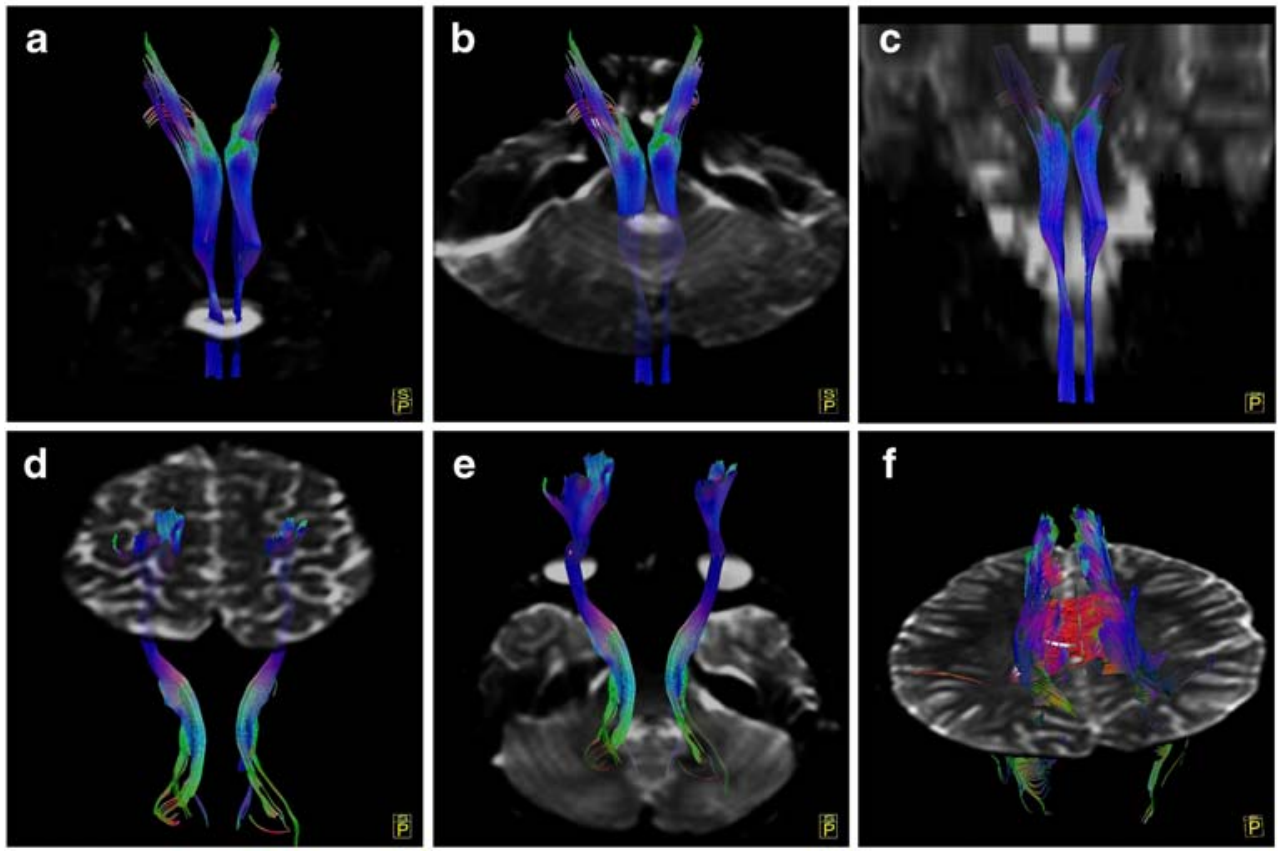

monaural auditory stimulation evokes predominantly contralateral activations [20]. In contrast, we observed that only left-sided monaural stimulation evoked bilateral activations in the primary auditory area, while right-sided monaural stimulation evoked no significant auditory activations. It is important to note that this observation was unlikely to have been an artefact because a similar neurophysiological observation has been made in two other previously reported patients [6]. Auditory stimulation of the right ear evoked no brainstem evoked potentials in either of these patients, while stimulation of the left ear evoked a complex bilateral response. There are two plausible explanations for this finding. First, the midline crossing fibers may be asymmet- rically affected and consequently one ear predominates and supplies bilateral auditory areas as seen in unilaterally deaf patients [21]. Second, although the crossing fibers are affected symmetrically, a monaural auditory system with bilateral auditory activations might be a physiological advantage as compared to a binaural but only unilateral auditory system. This physiological advantage might induce axonal sprouting or suppression. Similar changes in cortical activity have been reported in patients with strabismus or anisometropic amblyopia [22]. Unfortunately it is impossible to discriminate between these two possibilities based on the current investigation and this issue should be addressed in future studies designed to answer this question.
Fig. 5 Neurophysiology. a MEPs recorded over the abductor digiti $\mathrm{V}$ muscles elicited by magnetic stimulation over the right or left parietal region. The MEPs are dominantly ipsilateral to the stimulation side. b SSEPs recorded over the left central region (C3), right central region (C4) and the seventh cervical spine (C7). The evoked potentials are dominantly ipsilateral to the stimulation side (C3 left centroparietal region, $\mathrm{C} 4$ right centroparietal region, C7 seventh cervical vertebra)

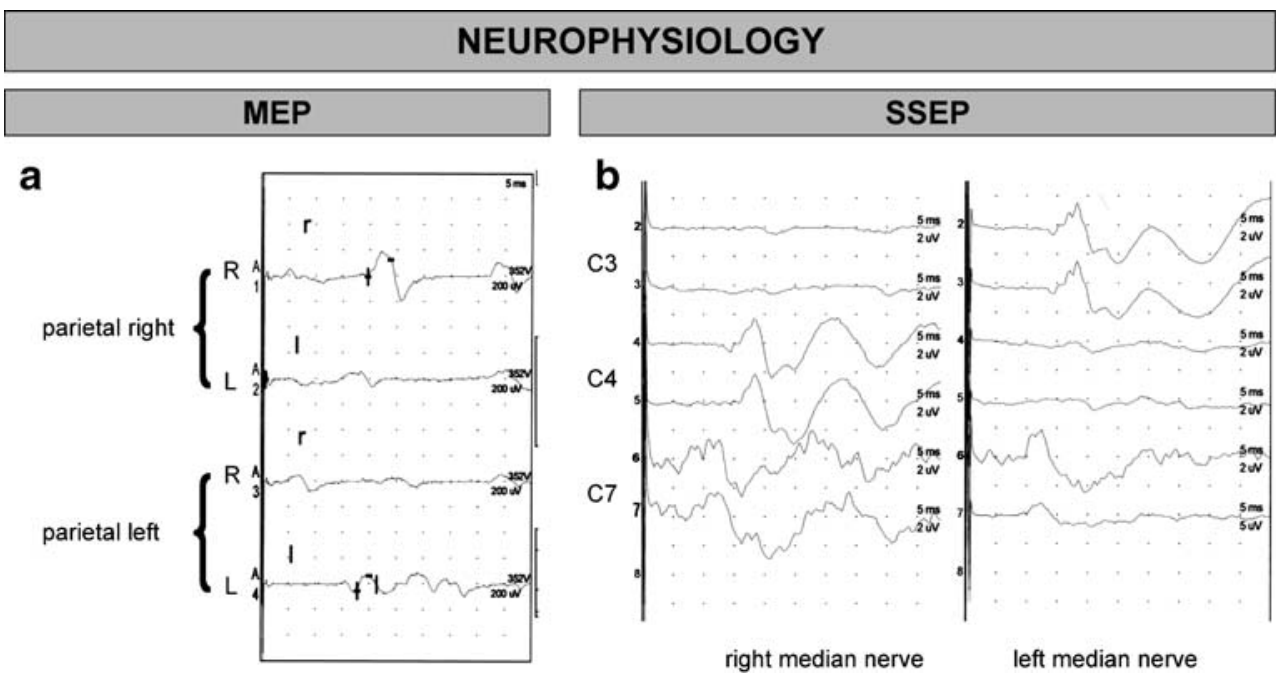

right median nerve 


\section{Conclusion}

Motor fMRI, DTI tractography and neurophysiology provided concordant ipsilateral sensorimotor findings as predicted in the rare disease HGPPS with absence of midline crossing fibers at the medulla. Auditory fMRI suggested that a monaural auditory system with bilateral auditory activations might be a physiological advantage as compared to a binaural yet only unilateral auditory system, in analogy to anisometropic amblyopia. Future, movinghead fMRI studies might show whether head movements compensating for horizontal gaze palsy activate the eyemovement network.

Conflict of interest statement We declare that we have no conflict of interest.

\section{References}

1. Dretakis EK, Kondoyannis PN (1974) Congenital scoliosis associated with encephalopathy in five children of two families. J Bone Jt Surg Am 56:1747-1750

2. Jen JC, Chan WM, Bosley TM, Wan J, Carr JR, Rub U, Shattuck D, Salamon G, Kudo LC, Ou J, Lin DD, Salih MA, Kansu T, Al Dhalaan H, Al Zayed Z, MacDonald DB, Stigsby B, Plaitakis A, Dretakis EK, Gottlob I, Pieh C, Traboulsi EI, Wang Q, Wang L, Andrews C, Yamada K, Demer JL, Karim S, Alger JR, Geschwind DH, Deller T, Sicotte NL, Nelson SF, Baloh RW, Engle EC (2004) Mutations in a human ROBO gene disrupt hindbrain axon pathway crossing and morphogenesis. Science 304:1509-1513

3. Jen J, Coulin CJ, Bosley TM, Salih MA, Sabatti C, Nelson SF, Baloh RW (2002) Familial horizontal gaze palsy with progressive scoliosis maps to chromosome 11q23-25. Neurology 59:432-435

4. Sicotte NL, Salamon G, Shattuck DW, Hageman N, Rub U, Salamon N, Drain AE, Demer JL, Engle EC, Alger JR, Baloh RW, Deller T, Jen JC (2006) Diffusion tensor MRI shows abnormal brainstem crossing fibers associated with ROBO3 mutations. Neurology 67:519-521

5. Bosley TM, Salih MA, Jen JC, Lin DD, Oystreck D, Abu-Amero KK, MacDonald DB, al Zayed Z, al Dhalaan H, Kansu T, Stigsby B, Baloh RW (2005) Neurologic features of horizontal gaze palsy and progressive scoliosis with mutations in ROBO3. Neurology 64:1196-1203

6. Amoiridis G, Tzagournissakis M, Christodoulou P, Karampekios S, Latsoudis H, Panou T, Simos P, Plaitakis A (2006) Patients with horizontal gaze palsy and progressive scoliosis due to ROBO3 E319K mutation have both uncrossed and crossed central nervous system pathways and perform normally on neuropsychological testing. J Neurol Neurosurg Psychiatry 77:1047-1053
7. MacDonald DB, Streletz LJ, Al-Zayed Z, Abdool S, Stigsby B (2004) Intraoperative neurophysiologic discovery of uncrossed sensory and motor pathways in a patient with horizontal gaze palsy and scoliosis. Clin Neurophysiol 115:576-582

8. Seifritz E, Esposito F, Hennel F, Mustovic H, Neuhoff JG, Bilecen D, Tedeschi G, Scheffler K, Di Salle F (2002) Spatiotemporal pattern of neural processing in the human auditory cortex. Science 297:1706-1708

9. Seifritz E, Di Salle F, Esposito F, Herdener M, Neuhoff JG, Scheffler K (2006) Enhancing BOLD response in the auditory system by neurophysiologically tuned fMRI sequence. Neuroimage 29:1013-1022

10. Talairach J, Tournoux P (1988) Co-planar stereotaxic atlas of the human brain. Thieme, New York

11. Genovese CR, Lazar NA, Nichols T (2002) Thresholding of statistical maps in functional neuroimaging using the false discovery rate. Neuroimage 15:870-878

12. Kakigi R (1986) Ipsilateral and contralateral SEP components following median nerve stimulation: effects of interfering stimuli applied to the contralateral hand. Electroencephalogr Clin Neurophysiol 64:246-259

13. Mori H, Fujishiro T, Hayashi N, Masutani Y, Aoki S, Ohtomo K, Aihara M, Wakakura M (2005) Partially uncrossed pyramidal tracts shown by tractography in horizontal gaze palsy and scoliosis. AJR Am J Roentgenol 184(3 Suppl):S4-S6

14. Berman RA, Colby CL, Genovese CR, Voyvodic JT, Luna B, Thulborn KR, Sweeney JA (1999) Cortical networks subserving pursuit and saccadic eye movements in humans: an FMRI study. Hum Brain Mapp 8:209-225

15. Petit L, Haxby JV (1999) Functional anatomy of pursuit eye movements in humans as revealed by fMRI. J Neurophysiol $82: 463-471$

16. Pierrot-Deseilligny C, Milea D, Muri RM (2004) Eye movement control by the cerebral cortex. Curr Opin Neurol 17:17-25

17. Zeki S, Watson JD, Lueck CJ, Friston KJ, Kennard C, Frackowiak RS (1991) A direct demonstration of functional specialization in human visual cortex. J Neurosci 11:641-649

18. Watson JD, Myers R, Frackowiak RS, Hajnal JV, Woods RP, Mazziotta JC, Shipp S, Zeki S (1993) Area V5 of the human brain: evidence from a combined study using positron emission tomography and magnetic resonance imaging. Cereb Cortex 3:7994

19. Ohlendorf S, Kimmig H, Glauche V, Haller S (2007) Gaze pursuit, 'attention pursuit' and their effects on cortical activations. Eur J Neurosci 26:2096-2108

20. Woldorff MG, Tempelmann C, Fell J, Tegeler C, GaschlerMarkefski B, Hinrichs H, Heinz HJ, Scheich H (1999) Lateralized auditory spatial perception and the contralaterality of cortical processing as studied with functional magnetic resonance imaging and magnetoencephalography. Hum Brain Mapp 7:49-66

21. Bilecen D, Seifritz E, Radu EW, Schmid N, Wetzel S, Probst R, Scheffler K (2000) Cortical reorganization after acute unilateral hearing loss traced by fMRI. Neurology 54:765-767

22. Norcia AM, Harrad RA, Brown RJ (2000) Changes in cortical activity during suppression in stereoblindness. Neuroreport 11:1007-1012 